\title{
Critical Remarks to Endoscopic Surgery for Endometrial Cancer and Sarcoma, Cervical and Ovarian Cancer Diagnosis and Treatment
}

ISSN: 2637-773X

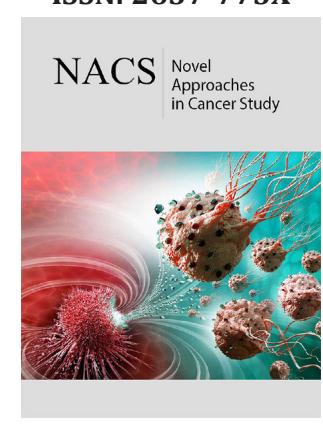

*Corresponding author: Mettler Liselotte, Department of Obstetrics and Gynecology, University Clinics of Schleswig Holstein, Kiel, Germany

Submission: 㘹 April 02, 2020

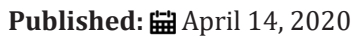

Volume 4 - Issue 3

How to cite this article: Mettler Liselotte, Ibrahim Alkatout. Critical Remarks to Endoscopic Surgery for Endometrial Cancer and Sarcoma, Cervical and Ovarian Cancer Diagnosis and Treatment. Nov Appro in Can Study. 4(3). NACS.000589. 2020. DOI: $10.31031 /$ NACS.2020.04.000589

Copyright@ Mettler Liselotte. This article is distributed under the terms of the Creative Commons Attribution 4.0 International License, which permits unrestricted use and redistribution provided that the original author and source are credited.

\author{
Mettler Liselotte* and Ibrahim Alkatout \\ Department of Obstetrics and Gynecology, Germany
}

\begin{abstract}
While endoscopic gynecological surgery as laparoscopy and hysteroscopy in all its variations of skills, like with single port entry, multiple port entry, conventional or robotically assisted has been universally completely accepted by the medical and patient community this is not at all yet a reality in oncological indications [1]. The article reviews the reality reality of Endoscopic surgery and its application in Gynecological Malignancy diagnosis and treatment focusing endometrial sarcoma, cervical and ovarian cancer.
\end{abstract}

General Development of Endoscopic Surgery in the Treatment for Gynecological Malignancies

We have observed an interesting sinus wave like development in the practice of oncologic gynecologic surgery, which started in the late 18th and early 19th century. This is easily demonstrated with the different treatment approaches to cervical cancer, starting from the Viennese school with Schauta and Wertheim, via Sakamoto, Dargent to Schneider, Bogges etc. $[1,2]$. The first critical voices against endoscopic surgery of even benign ovarian tumors came up in the early 1980ties till tissue extraction with endobags was generally introduced and used. The spread of tumor cells by $\mathrm{CO} 2$ gas, by washing procedures with saline solution as well as port site metastases are still not completely clear.

With the world wide acceptance of the advantages of endoscopic surgery compared to vaginal and open surgery already since a good 30 years also oncological procedures with identical outcome to the conventional open surgical procedures over the last 100 years established gynecological surgical procedures were reported. Particularly for early endometrial cancers identical numbers of lymph nodes and disease free periods and overall survival rates of patients were found in randomizes studies. Also publications on early stages of cervical cancer treatments [2] increased, particularly since the introduction of Robot assisted endoscopic surgery. Tissue extraction and lymph node extraction, including the recent visualization with ICG, were performed in a multitude of ways [3,4].

In the following we discuss 3 specific issues in the treatment of gynecologic oncologic cases focusing on a possible benefit of laparoscopy as part of the complex treatments.

\section{Tissue Morcellation and the Sarcoma Thread for Endometrial Adenocarcinoma and Leiomyosarcoma}

In 2014 concerns for tissue morcellation as a reason for "sarcoma spreading" evoked a worldwide discussion. Uterine fibroids are benign neoplasms, affecting 20-40\% of women during their reproductive years [1]. When laparoscopic or hysteroscopic operations are performed today electromechanical morcellation is used, which allows removal of uterine fibroids and uterine tissue through port sites. However, morcellation procedures can potentially cause intraoperative dissemination of pathological uterine tissue into the abdominal cavity, especially in unsuspected malignant cases, which may lead to a spreading of the disease and may have negative clinical consequences.

After early developments of manual morcellators to solve the problem of tissue extraction in Kiel Germany around 1991 [1] the serrated edged macro-morcellator (SEMM) working 
electronically by punching out tissue cylinders of 1, 1, 5 and $2 \mathrm{~cm}$ in diameter, in length measuring up to $10-20 \mathrm{~cm}$ specimen particles was developed. Karl Storz then developed an even more competitive morcellator in 2007 called the Rotocut G1 morcellator. In comparison to the existing Sawalhe model, in the Rotocut G1 device accomplished significantly shorter morcellation time and duration of anesthesia. The Gynecare Morcellex tissue morcellator developed by Ethicon, Inc. is another popular power morcellator. It was withdrawn from the market by Johnson and Johnson after a statement discouraging the use of power morcellators was released by the FDA (See "Updated FDA Recommendations").

Till today, being aware of the advantages of tissue morcellation the biggest concern in regards to dissemination of tissue during morcellation is the inadvertent dissemination of malignancy.

\section{Endometrial adenocarcinoma}

If morcellation of endometrial adenocarcinoma can cause cancerous tissue to be spread throughout the abdomen and lead to possible upstaging of an existing malignancy this does appear as a maximal thread. Morcellation of endometrial adenocarcinoma can usually be avoided by appropriate pre-operative evaluation with endometrial biopsy or dilation and curettage; however, this is not always accurate. Studies have shown a discrepancy of $10-16 \%$ in histologic diagnosis with endometrial biopsy or curettage when compared to hysterectomy. A recent European evaluation showed a concordance rate of only $62 \%$ and $67 \%$ of endometrial biopsy and curettage (respectively) when compared to hysterectomy.

\section{Leiomyosarcoma}

Since the beginning of introducing tissue morcellation into gynecology it was strongly advised to exclude any case of unclear preoperative pathology from morcellation. Of greatest concern is the inadvertent morcellation of a leiomyosarcoma as leiomyosarcoma is the most common malignant nonepithelial tumor of the uterus representing $1-2 \%$ of all uterine malignancies. It is an aggressive malignancy with 5-year survival rates of $18.8 \%$ to $65 \%$. Particularly women in the perimenopausal years are affected. Their median age of 52 years and sarcomas are very rare in women below the age of 40 years. Mostly this malignancy reveals a rapidly growing uterus; however, because it is uncommon, only $0.23 \%$ of patients with this finding will have leiomyosarcoma. Preoperative diagnosis is challenging because the symptoms and the clinical appearance associated with leiomyosarcoma are nearly identical to benign leiomyoma. Magnetic resonance imaging (MRI) with diffusion- weighted imaging and PET/CT with F-FDG have both been evaluated in the pre-operative diagnosis of leiomyosarcoma; neither has been sufficiently proven to offer accurate diagnosis. Utilizing serum lactate dehydrogenase (LDH) and its isoenzymes, especially isozyme type 3 , has also been utilized to distinguish leiomyosarcoma from benign leiomyoma and is a promising technique. There are currently 9 studies in the literature on unsuspected leiomyosarcomas in patients who had a hysterectomy or myomectomy for presumed benign disease. The rate of leiomyosarcoma in these studies ranges from $0-0.49 \%$ with the average being $0.18 \% 169$. The FDA recently released a statement quoting the incidence to be 1 per 350 or $0.29 \%$. This incidence has been challenged by Pritts et al. at the FDA meeting. Her more extensive evaluation of the literature was correlated to a much lower risk of leiomyosarcoma. Morcellating these unsuspected malignancies can result in upstaging and a worse prognosis. Due to these controversial findings and reports, the FDA put out a statement discouraging the use of power morcellators, citing safety concerns, mostly the inadvertent dissemination for occult uterine cancer in patients undergoing hysterectomy and myomectomy for presumed leiomyomata [1].

\section{Contained morcellation - Does it diminish the risk?}

At the University Department Obstetrics \& Gynecology in Kiel, Germany we included into our retrospective "morcellation evaluation study" patient's data from 2003 to 2015 [5]. Proportion confidence intervals were calculated using the Wilson method. Continuous variable confidence intervals were calculated. Between 2003 and 2015, 2269 women received a preoperative diagnosis of presumed benign uterine fibroids. Of these, 938 (41.3\%) women had myomectomies and 1331 (58.7\%) women had hysterectomies, only one patient was postoperatively diagnosed as having endometrial stromal sarcoma (ESS) based on a pathohistologic analysis; the patient was aged 48 years and her BMI was 26.5 $(\mathrm{kg} / \mathrm{m} 2)$ at the time of diagnosis, and she reported hyper and polymenorrhea. The patient's medical history was otherwise unremarkable. Ultrasound examination showed a hypoechogenic lesion $(8.5 \mathrm{~cm})$ with a clear borderline on the left posterior wall of the uterus. Physical examination of the external genitalia, vagina and cervix showed no abnormalities. Pelvic examination revealed a uniformly enlarged uterus, and no adnexal masses were palpated. The primary diagnosis was symptomatic transmural leiomyoma. The patient underwent a laparoscopic subtotal hysterectomy (LSH) with morcellation. The final pathohistologic results detected ESS, and the specimen weighed $294 \mathrm{~g}$. Immunohistochemical analysis showed the following results: $\operatorname{CD} 10(+)$, desmin $(+), \operatorname{SMA}(-)$, actin( $(-)$ and 5\% Ki67(+). Furthermore, immunohistochemical assays for ERs, PRs, and p53 showed the following results: ER (+++), PR (+++) and p53 (-). Two weeks after the initial surgery, a laparotomic removal of cervical stump with bilateral salpingo-oophorectomy and an omentectomy was performed. The final pathologic report described no signs of any metastatic lesions.

Six patients who were diagnosed preoperatively as having suspected uterine sarcoma were treated and followed up according to the treatment protocol for uterine sarcomas. The four women diagnosed as having uterine leiomyosarcoma (ULMS) were postmenopausal, and their dominant presenting symptom was postmenopausal bleeding. The mean age of the women with ULMS at the time of diagnosis was $73.7 \pm 3.9$ years (range, 68-79 years), and their mean BMI was $27.2 \pm 3.7 \mathrm{~kg} / \mathrm{m} 2$ (range, $22.2-30.8 \mathrm{~kg} / \mathrm{m} 2$ ).

The frequency of unsuspected ULMS, HGUS, and ERMS was 0/2269. For ESS, it was 1/2269 [0.044\%, 95\% CI: (0.001-0.25)] among the women in this study who underwent myomectomies and hysterectomies for the treatment of presumed benign uterine fibroids. The total number of patients with uterine sarcoma was 
seven during the twelve-year period. Out of them, 6 were diagnosed through biopsy prior and during their larger surgical intervention, Only one ESS was diagnosed after a laparoscopic subtotal hysterectomy [5].

\section{How are we supposed to handle tissue extraction today?}

In bag morcellation certainly is advised for all morcellation procedures, although we do not know for sure if already the enucleation process or $\mathrm{CO} 2$ are presenting a risk factor in unknown malignancy cases treated by laparoscopy. Our advise at present is: let us follow our knowledge and intuition of te individual case, discuss details with the patient, advantages and disadvantages, and wait for further international final conclusions concerning tissue morcellation issue. Once the diagnosis can be made from homogenized tissue in a bag, genetically analyzed after the surgery, everything will be easier and a homogenization in a bag will be self understood.

\section{Cervical Cancer}

\section{Developments of cervical cancer surgery, open, conventional laparoscopic and robotically assisted laparoscopic}

Surgical treatment for this disease goes back to Schauta and Wertheim [6,7] and to a multitude of serious and very dedicated gynecological surgeons all over the world. Even the specific pelvic laparoscopic anatomy has been described in detail [8-10]. For early-stage disease, surgical removal of the uterus remains the primary treatment and has the greatest effect on long-term survival. As abdominal ("open") radical hysterectomy is associated with complications, including a risk of lymphedema in the legs and bladder and sexual dysfunction since 30 years the development of minimally invasive techniques for cervical cancer surgery increased steadily. Despite a paucity of randomized trials, retrospective data suggesting superior surgical and similar oncologic results led to widespread acceptance of minimally invasive radical hysterectomy. Laparoscopy-based and robotic techniques are currently challenging methods of performing radical hysterectomies worldwide [11].

\section{Safety, surgical results, recurrence and survival rates}

Lately in 2018 protest waves turn against laparoscopic treatment of Cervical cancer as cervical cancer knowing that it still is one of the most fatal causes of cancer-related death in females. Ramirez and colleagues [12,13] now report in the New England Medical Journal the results of the Laparoscopic Approach to Cervical Cancer (LACC) Trial, a phase 3 trial comparing minimally invasive (laparoscopic or robotic) radical hysterectomy with open radical hysterectomy in women with early-stage cervical cancer. Midway through the trial, the data and safety monitoring committee called for early closure of the trial after an interim analysis revealed a disease-free survival rate at 4.5 years that was lower with minimally invasive surgery than with open surgery $(86.0 \%$ vs. $96.5 \%$; difference, -10.6 percentage points) and a lower 3-year rate of overall survival (93.8\% vs. 99.0\%; hazard ratio for death, 6.00). A companion population- based study by Melamed and collaborators, showed similar survival trends in a two-part analysis [13].
The first analysis, which used propensity-score weighting, showed that women with early-stage cervical cancer who had been treated at Commission on Cancer-accredited hospitals with minimally invasive radical hysterectomy had a lower rate of overall survival within 4 years after diagnosis than those who had been treated with open radical hysterectomy $(90.9 \%$ vs. $94.7 \%, \mathrm{P}=$ 0.002). The second analysis, a time-interrupted study of a similar population that used data from the Surveillance, Epidemiology, and End Results program database, showed a progressive decrease in the 4 -year relative survival rate by $0.8 \%$ per year that coincided with the initial period of adoption of robot-assisted surgery in the United States (2007-2010) ( $\mathrm{P}=0.01$ for change of trend). Taken together, the LACC Trial and the epidemiologic study call into question the equivalency of cancer outcomes with open as compared with minimally invasive radical hysterectomy for cervical cancer.

The population-based study by Melamed et al. [13] and the LACC Trial had similar study populations and similar survival outcomes. Furthermore, this randomized trial involved 33 centers in 13 countries and the participation of high-volume cervicalcancer surgeons. The surgical-volume requirement and quality assessment of the participating surgeons' skills and postoperative outcomes distinguish this trial from previous phase 3 cervicalcancer trials and may have contributed to the more favorable outcomes with open radical hysterectomy than those observed in the previous trials [14-18]. Curiously, all cancer recurrences in the LACC Trial were clustered at 14 of the 33 participating cancer centers, which raises questions about whether those centers enrolled more patients, enrolled them earlier, or had unique patient or surgeon factors. In addition, the cumulative incidence of locoregional recurrence was surprisingly higher in the minimally invasive surgery group than in the open-surgery group.

The use of uterine or cervical manipulators and carbon dioxide (CO2) gas in minimally invasive radical hysterectomies is postulated to encourage local tumor spread. Yet, statistics on manipulator usage were not presented in the LACC Trial, and data from randomized trials are mixed regarding the contributions of CO2 pneumoperitoneum to the promotion of tumor recurrence (with the latter factor more likely to contribute to abdominal and port-site metastases than to loco- regional disease spread). Other factors, such as surgical technique, degree of procedural radicality, and peritoneal immunity, may also contribute. Ad hoc studies that evaluate manipulator use and its association with recurrence as well as surgical techniques to minimize cervical tumor spread may help clarify these questions.

Although the results of the LACC Trial and the epidemiologic study are powerful, scientific scrutiny demands consideration of potential study-design or study-conduct issues that may affect outcomes unexpectedly. Surgical trials are difficult to conduct and pose particular practical and methodologic challenges. One concern is the early closure of the trial, with enrollment of $85 \%$ of planned participants and somewhat reduced power for the primary outcome; however, the trial statistics are valid on the basis of the wide margin of difference and nonoverlapping 95\% confidence intervals in the disease-free survival rates of the surgical cohorts. 
Additional limitations that may warrant future study include the imperfect assessments of cervical- cancer stage, the lack of follow-up data and missing data in select patients, the lack of data regarding patient race and ethnic group, non- standardization of adjuvant treatment, and non- performance of central pathology review. How- ever, for most outcomes, these trial shortcomings appeared balanced between the two groups. Limitations of the epidemiologic study included the retrospective data sets, the heterogeneous methods of cancer staging, and the fact that the time frame of the study coincided with the earlier part of the roboticsurgery learning curve for radical hysterectomy.

\section{Does minimally invasive radical hysterectomy in cervical-cancer treatment come to a halt now?}

Not completely but it tells us to reflect on reasons why this happened. Although the data are alarming, select patient subgroups may still benefit from a less invasive approach. No patients with stage IA2 disease and only one with stage IB1, grade 1, disease had a recurrence in the LACC Trial. In addition, patients with a tumor size of less than $2 \mathrm{~cm}$ did not have worse outcomes with minimally invasive surgery than with open surgery in either study. Until further details are known, however, surgeons should proceed cautiously, counsel their patients regarding these collective study results, and assess each woman's individual risks and benefits with respect to minimally invasive as compared with open radical hysterectomy.

The whole issue may be a surgical problem of laparoscopically not being radical enough and observing the lymph drainage as much as really necessary. Another factor might be the usage of intra uterine manipulators, which should not be used; a vaginally prepared cervical manchetta or cuff at the beginning of the radical hysterectomy with lymph adenectomies closes up the tumor in a natural tissue bag and may stop a possible tu $\neg$ mor spread during the surgery. The German team of Koehler et al. [19] already came up with a new study presenting the advantages of the protective manchetta surgery performed vaginally around the cervical cancer at the beginning of the surgical minimal invasive procedure. This technique was always practiced already by Dargent [2] and his team. This German team evaluated 1952 consecutive patients with cervical cancer treated between 1994 and 2018 (stages 1A1 -1B1 coming up with the conclusions that vaginally assisted laparoscopic radical hysterectomy was oncologically safe in patients with early cervical cancer in their retrospective study, it avoids the use of manipulator and potentially prevents tumor spillage.

From 1994 to 2005 they used the technique of laparoscopic assisted radical vaginal hysterectomy for combined laparoscopicvaginal radical hysterectomy with predominant vaginal resection of parametria. Despite encouraging oncologic results, [16] they had to modify this approach due to a peri- and post-operative urologic complication rate of $>10 \%$ and the fact that it was very difficult to spread this approach. From 2005 up to now the technique of vaginal-assisted laparoscopic radical hysterectomy14 has been used. Both techniques have already been described in detail $[4,14]$. In both techniques the vaginal creation of a tumor covering vaginal cuff and the strict avoidance of use of any uterine manipulator is mandatory. The key differences of vaginal-assisted laparoscopic radical hysterectomy compared with standard minimally invasive radical hysterectomy are based on the formation of the vaginal cuff. In accordance with guidelines (ESGO) to avoid combined morbidity of radical hysterectomy and adjuvant chemoradiation, their surgery has always been abandoned in patients with positive lymph nodes on frozen section, and primary chemoradiation or radiotherapy performed [17]. The oncologic and survival outcomes of vaginal laparoscopic radical hysterectomy were similar to those of open radical hysterectomy in the LACC trial.

It looks that the time has come for a next randomized control trial comparing recurrence rates and long time survival rates of early cervical endoscopic cancer surgery with the for $\neg$ mation of a vaginal cuff as originally done with the Schauta technique, performed by many gynecological cancers surgeons and nicely described by Koehler Ch. et al [19] to open laparotomy radical surgery.

\section{Ovarian Cancer}

\section{Facts and reality}

According to the National Cancer Network NCCN clinical practice guidelines in oncology for ovarian cancer in version 2, laparoscopy can accurately diagnose the extensiveness of disease. When performed after standard diagnostic work-up less women had unsuccessful debulking surgery and therefore resulting in less morbidity [20]. The initial treatment for stage I ovarian cancer is surgery to remove the tumor. Most often the uterus, both fallopian tubes, and both ovaries are removed (a hysterectomy with bilateral salpingo-oophorectomy). The treatment after surgery depends on the sub-stage of the cancer.

\section{Stages IA and IB (T1a or T1b, N0, M0): The treatment after surgery depends on the tumor grade).}

I. For grade 1 (low grade) tumors, most women don't need any treatment after surgery. Women who want to be able to have children after treatment might be given the option of having an initial surgery that removes only the ovary containing the cancer along with the fallopian tube on the same side.

II. For grade 2 (high grade) tumors, patients are either watched closely after surgery without further treatment, or they are treated with chemotherapy (chemo). The chemo used most commonly is carboplatin and paclitaxel (Taxol) for 3-6 cycles, but cisplatin can be used instead of carboplatin, and docetaxel (Taxotere) can be used instead of paclitaxel.

III. For grade 3 (high grade) tumors, the treatment usually includes the same chemotherapy that is given for grade 2 Stage IA and IB cancers.

Stage IC (T1c, N0, M0): Standard surgery to remove the cancer is still the first treatment. After surgery, chemo is recommended, usually with 3 to 6 cycles of treatment with carboplatin and paclitaxel.

Stage I fallopian tube and primary peritoneal cancers are treated the same way as stage I ovarian cancer. 
Stage II cancers: For stage II (including IIA and IIB) cancers, treatment starts with surgery for staging and debulking. This includes a hysterectomy and bilateral salpingo-oophorectomy. After surgery, chemo is recommended for at least 6 cycles. The combination of carboplatin and paclitaxel is used most often. Some women with stage II ovarian cancer are treated with intraperitoneal (IP) chemotherapy instead of intravenous (IV) chemotherapy. Stage II fallopian tube and primary peritoneal cancers are also treated with surgery for staging and debulking, followed by chemo.

Stage III cancers: Stage III cancers (including IIIA1, IIIA2, IIIB, and IIIC) are generally treated similarly to stage II cancers. First, the cancer is surgically staged and the tumor is debulked (like stage II). The uterus, both fallopian tubes, both ovaries, and omentum (fatty tissue from the upper abdomen near the stomach and intestines) are removed. The surgeon will also try to remove as much tumor as possible. The goal is to leave behind no visible tumor or no tumor larger than $1 \mathrm{~cm}$. When this goal is reached, the cancer is said to have been optimally debulked. Sometimes tumor is growing on the intestines, and in order to remove the cancer, part of the intestine will have to be removed. Sometimes pieces of other organs (like the bladder or liver) may also have to be removed to take out the cancer. The smaller the remaining tumor, the better the outlook will be. After recovery from surgery, combination chemo is given. The combination used most often is carboplatin (or cisplatin) and a taxan, such as paclitaxel (Taxol), given IV for 6 cycles. The targeted drug bevacizumab (Avastin) might be given along with chemo as well. Another option is to give intra-abdominal (intraperitoneal or IP) chemo along with intravenous (IV) chemo, after surgery. IP chemo is usually only considered if the cancer was optimally debulked - it may not work as well if a lot of tumor is left in the abdomen

After surgery, and during and after chemo, blood tests checking for the CA-125 tumor marker will be done to see how well the treatment is working. A CT scan, PET-CT scan, or MRI might also be done.For women who are not healthy enough to have full staging and debulking surgery, chemo might be given as the first treatment., surgery to debulk the cancer may be done, often followed by more chemo. Most often, 3 cycles of chemo are given before surgery, with at least 3 more after surgery Giving chemo before surgery is also sometimes an option for some women with advanced cancers that aren't likely to be optimally debulked if surgery is done first [21]. Maintenance therapy: If the cancer appears to be gone after the initial treatment, additional chemotherapy can be applied This is called maintenance therapy. It is aimed at killing any cancer cells that were left behind after treatment but are too small to be seen on tests. The goal of maintenance therapy is to keep the cancer from coming back after treatment (Paclitaxel, Pazopanib, Niraparib, Olaparib). But since the studies so far show that maintenance therapy does not necessarily help to live longer and may cause more side effects, this is still being studied in clinical trials.

Stage IV cancers: In stage IV, the cancer has spread to distant sites, like the liver, the lungs, or bones. These cancers are very hard to cure with current treatments, but they can still be treated. The goals of treatment are to help patients feel better and live longer.
Stage IV can be treated like stage III, with surgery to remove the tumor and debulk the cancer, followed by chemo (and possibly the targeted drug bevacizumab [Avastin]).

\section{Role of Laparoscopy in diagnosing extensiveness of ovarian cancer}

Why is improving the diagnosis of extensiveness of ovarian cancer important?

Ovarian cancer is a disease with a high-mortality (death) rate. Many women (75\%) are diagnosed when their disease is already at an advanced stage and 140,000 women die of this disease each year worldwide. Treatment consist of debulking surgery (removal of as much of the tumor as possible during an operation called a laparotomy - normally through a long vertical cut on the abdomen) and six cycles of chemotherapy. The order in which these two treatments are given depends on the extensiveness of disease (how widespread) and on the general health of the patient. The goal of debulking surgery is to remove all visible tumor or at least to leave no residual tumor deposit bigger than $1 \mathrm{~cm}$ in diameter. When the diagnostic evaluation suggests that the goal of debulking surgery could not be achieved, initial treatment may be three cycles of chemotherapy to first shrink the tumor, followed by debulking surgery and then further chemotherapy to complete the course of six cycles of chemotherapy.

To diagnose the extensiveness of disease by physical examination, ultrasonography, abdominal computed tomography (CT scan), and measurement of serum tumor (blood) markers are performed. An incorrect diagnosis could result in women having unsuccessful primary debulking surgery. An interesting review aimed at reviewing if laparoscopy is accurate in predicting whether a woman can be successfully operated to remove of all visible tumor or at least to leave no tumor deposits larger than $1 \mathrm{~cm}$. If so, this could help to avoid operating on those women who would be better treated with chemotherapy first. This review included a total of 18 relevant studies, 11 of which were added for this update, and looked at 14 groups of women. In total 1563 women underwent a laparoscopy to evaluate the extensiveness of disease in the abdomen. Two studies concluded that laparoscopy was good at identifying those women in whom optimal debulking surgery was not feasible (with tumor deposits $>1 \mathrm{~cm}$ left after surgery) (low false positive rate for laparoscopy) and in all women the diagnosis was correct. However, even after a laparoscopy had suggested that optimal debulking surgery was feasible, some women had suboptimal primary debulking surgery where tumor deposits of $>1 \mathrm{~cm}$ were left. For every 100 women referred for primary debulking surgery after laparoscopy, between four and 46 will be left with visible residual tumor.

\section{Discussion, Conclusions and Vision for all 3 Discussed Gynecological Cancer Treatments}

Modern gynecological surgery is only 150 -200 years old. As access routes we have the vaginal pathway, the transabdominal entry, the gastro intestinal or transvesical entry into the abdomen and image guided entries with intercommunications. 
By hysteroscopy we can take advantage of a direct entry under vision into the uterine cavity. By laparoscopy the direct entry is under $\mathrm{CO} 2$ expansion of the abdominal cavity by the conventional and the robotic way. Surgery includes diagnosis and treatment in benign and malignant masses and cannot be separated from each other. Knowledge of clinical anatomy forms the basis for all surgical procedures. Endoscopic and image guided surgery are already well established procedures that are developed for benign and malignant surgery. Particularly for the treatment of malignancies they have to be specifically evaluated and compared to existing well established procedures to really show us the best way of treating our patients. We are, however, sure that better vison, more precision and less tremor in resecting tissue can only improve the outcome if resection is the issue.

It took about 50 years for endoscopic surgery to be integrated into the education of medical student programs, into nearly every operative set up and institution around the world. Kurt Semm's wish became true is implied today nearly everywhere and our vision of teaching and studying many still underlining shortcomings sees this surgery together with imaging technologies to lead the correct way for the future.

Surgical specialties have separated from each other for good reasons. Even the specialties have become so specific that we cannot learn everything and can only dominate well some smaller fields. However, a healthy cooperation and exchange of ideas between general surgeons, urologist, gynecologists, neurologists and orthopedic surgeons etc. can only carry benefit for one or the other. Neuropelveology as new technique named by Marc Possover shows us current important fields of interaction [22].

In 2016 we founded the Kurt Semm Center for Endoscopic Conventional and Robotic Surgery Kiel, Germany, as an interdisciplinary center for urologists, general surgeons, gynecologist, etc. We intend to keep on going strong following the guide lines established by the different professional societies in our country and worldwide, while keeping the interest of our patients and doctors focused and based on new clinical and research findings. By many clever people surgery is not recognized or estimated as science, however, particularly in the treatment of malignancies it still is the most effective tool that we have available and this has to be cherished and recognized. In this "reality and dream" endoscopic surgeons in all surgical specialties dealing with malignancies keep on serving our patients with best intensions in all our actions.

\section{References}

1. Alkatout I, Mettler L (2019) Practical manual for laparoascopic and hysteroscopic surgery. Kiel School of Gynecological Laparoscopic Surgery, Yaypee Brothers, Hyderabad, India, p.1500.

2. Dargent D (1987) A new future for schauta's operation through presurgical retroperitoneal pelviscopy. European Journal of Gynaecological Oncology 8: 292-296.

3. Bray F, Ferlay J, Soerjomataram I, Siegel RL, Torre LA, et al. (2018) Global cancer statistics 2018: GLOBOCAN estimates of incidence and mortality worldwide for 36 cancers in 185 countries. CA Cancer J Clin 68(6):394424.
4. Conrad LB, Ramirez PT, Burke W, Naumann RW, Ring KL, et al. (2015) Role of minimally invasive surgery in gynecologic oncology: An updated survey of members of the society of gynecologic oncology. Int J Gynecol Cancer 25(6): 1121-1127.

5. Mettler L, Abdusattarova K (2017) Concerns for "tissue morcellation" The thread of sarcomas. MOJ Womens Health 5(2): 209-211.

6. Schauta F (1908) Die erweiterte vaginale Totalexstirpation des Uterus bei Kollumkarzinom. Wien Leipzig: Josef Safár.

7. Wertheim E (1911) Die erweiterte abdominale Operation bei carcinoma colli uteri (auf Grund von 500 Fällen). Berlin, Wien: Urban und Schwarzenberg.

8. Dargent D, Martin X, Sacchetoni A, Mathevet P (2000) Laparoscopic vaginal radical trachelectomy: A treatment to preserve the fertility of cervical carcinoma patients. Cancer 88(8): 1877-1882.

9. Magrina JF, Pawlina W, Kho RM, Magtibay PM (2011) Robotic nervesparing radical hysterectomy: feasibility and technique. Gynecologic Oncology 121(3): 605-609.

10. Shailesh P, Nanda S, Parikh K (2019) Laparoscopic pelvic anatomy in females. Applied Surgical Principles.

11. Comprehensive Cancer Network (2017) NCCN clinical practice guidelines in oncology: Cervical cancer (version I.2018).

12. Ramirez PT, Frumovitz M, Pareja R, Lopez A, Vieira M, et al. (2018) Minimally invasive versus abdominal radical hysterectomy for cervical cancer. N Engl J Med 379(20): 1895-1904.

13. Melamed A, Margul DJ, Chen L, Keating NL, Del Carmen MG, et al. (2018) Survival after minimally invasive radical hysterectomy for early-stage cervical cancer. N Engl J Med 379(20): 1905-1914.

14. Cook JA (2009) The challenges faced in the design, conduct and analysis of surgical randomized controlled trials. Trials 10: 9.

15. Delgado G, Bundy B, Zaino R, Sevin BU, Creasman WT, et al. (1990) Prospective surgical- pathological study of disease-free interval in patients with stage IB squamous cell carcinoma of the cervix: A gynecologic oncology group study. Gynecol Oncol 38(3): 352-357.

16. Rotman M, Sedlis A, Piedmonte MR, Bundy B, Lentz SS, et al. (2006) A phase III randomized trial of postoperative pelvic irradiation in stage IB cervical carcinoma with poor prognostic features: follow-up of a gynecologic oncology group study. Int J Radiat Oncol Biol Phys 65(1): 169-176.

17. Peters WA III, Liu PY, Barrett RJ II, Stock RJ, Monk BJ, et al. (2000) Concurrent chemo- therapy and pelvic radiation therapy compared with pelvic radiation therapy alone as adjuvant therapy after radical surgery in high-risk early-stage cancer of the cervix. J Clin Oncol 18(8): 16061613.

18. Mo X, Yang Y, Lai H, Xiao J, He K, et al. (2014) Does carbon dioxide pneumo- peritoneum enhance wound metastases following laparoscopic abdominal tumor surgery? A meta-analysis of 20 randomized control studies. Tumour Biol 35(8): 7351-7359.

19. Koehler Chr, Herman Hertel J, Marnitz S, Marnitz P, Mallmann G, et al. (2019) Laparoscopic radical hysterectomy with transvaginal closure of vaginal cuff - a multicenter analysis. International Journal of Gynecological Cancer 29(5): 845-850.

20. Ovarian Cancer: National Comprehensive Cancer Net- work. NCCN clinical practice guidelines in oncology: ovarian cancer version 2.2018

21. Moore K, Colombo N, Scambia G, Kim BG, Oaknin A, et al. (2018) Maintenance olaparib in patients with newly diagnosed advanced ovarian cancer. The New England Journal of Medicine 379: 2495-2505.

22. Possover M (2020) Neuropelveology: New possibility for the diagnosis and treatment of neuropathic pelvic pain. Endometriosis. 
For possible submissions Click below:

\section{Submit Article}

